\title{
Antoine Bevort, Annette Jobert, Michel Lallement, Arnaud Mias (dir.), Dictionnaire du travail
}

Paris, Presses universitaires de France, collection « Quadrige dicos poche ", 2012

\section{André Grelon}

\section{(2) OpenEdition}

\section{Journals}

Édition électronique

URL : http://journals.openedition.org/travailemploi/6028

DOI : $10.4000 /$ travailemploi.6028

ISSN : 1775-416X

Éditeur

DARES - Ministère du Travail

\section{Édition imprimée}

Date de publication : 15 juin 2013

Pagination : 91-93

ISSN : 0224-4365

\section{Référence électronique}

André Grelon, « Antoine Bevort, Annette Jobert, Michel Lallement, Arnaud Mias (dir.), Dictionnaire du travail », Travail et Emploi [En ligne], 134 | avril-juin 2013, mis en ligne le 01 avril 2013, consulté le 22 septembre 2020. URL : http://journals.openedition.org/travailemploi/6028 ; DOI : https://doi.org/ 10.4000/travailemploi.6028

Ce document a été généré automatiquement le 22 septembre 2020.

(c) Direction de l'animation de la recherche, des études et des statistiques (Dares) 


\section{Antoine Bevort, Annette Jobert, Michel Lallement, Arnaud Mias (dir.), Dictionnaire du travail}

Paris, Presses universitaires de France, collection «Quadrige dicos poche », 2012

\section{André Grelon}

\section{RÉFÉRENCE}

Antoine Bevort, Annette Jobert, Michel Lallement, Arnaud Mias (dir.), Dictionnaire du travail, Paris, Presses universitaires de France, collection « Quadrige dicos poche », 2012, 880 p.

Cinquante ans exactement après la parution du Traité de sociologie du travail sous la direction de Georges Friedmann et Pierre Naville (1962, Armand Colin, 2 tomes, 468 et 439 p.), les Presses universitaires de France offrent au public une œuvre aussi ambitieuse avec ce Dictionnaire du travail, premier du genre en France. Sans systématiser outre mesure, il peut être intéressant de regarder le contenu de ce nouvel opus en allant consulter ce qu'on pouvait lire dans cette première étude collective sur le travail. Les directeurs du Traité avaient rassemblé vingt-sept auteurs (dont trois femmes), le Dictionnaire s'appuie sur les textes de 139 contributeurs (avec $30 \%$ de femmes). Malgré son titre, le Traité était un travail pluridisciplinaire, ses directeurs exposant dans l'avant-propos la nécessité, par la variété des problèmes traités, de collaborations diverses : un économiste, un démographe, deux ethnologues, un médecin du travail, un conseil en organisation, un statisticien, un juriste, et un psychologue. On retrouve cette même indispensable diversité dans le Dictionnaire, même si les sociologues se taillent la part du lion, constituant $63 \%$ des auteurs. Les économistes sont loin derrière (10\%), les historiens sont encore moins nombreux (6\%) avec les politistes (5\%). On trouve aussi cinq juristes, quatre ergonomes, trois gestionnaires, deux psychologues, un 
anthropologue, une linguiste, un philosophe, etc. Seules disciplines de sciences sociales non convoquées dont on aurait pu penser qu'elles avaient leur place dans une telle somme: la géographie et la démographie. À l'exception d'une dizaine de collègues suisses, canadiens et belges et d'un Britannique, les contributeurs sont français, inscrits dans des structures académiques; cinquante-quatre universitaires, trente-deux chercheurs au $\mathrm{CNRS}^{1}$, dix au $\mathrm{Cnam}^{2}$, neuf à Sciences-Po, quatre à l'EHESS ${ }^{3}$, trois à l'Insee ${ }^{4}$, etc., tous spécialistes incontestés du domaine qu'il leur a été demandé de traiter.

2 Le dictionnaire se compose de 141 entrées, soit des textes de taille homogène (de cinq à sept pages). Chacun est suivi d'une bibliographie focalisée et d'une petite liste de corrélats, avant la signature de l'auteur. On va de A avec « action collective » à V pour «ville ». Curieusement, la lettre L n'est pas mentionnée. On peut penser qu'on aurait pu y trouver des analyses sur les langues, les législations, la liberté, le libre-échange, le lien social, les loisirs ou les luttes sociales notamment. Il est vrai que même un volume de cette ampleur ne peut tout dire et que ce dictionnaire est muni d'un précieux index thématique (dit index rerum) de pas moins de 683 items où le lecteur intrigué peut retrouver à peu près tous ces termes pour la lettre L, à l'exception de libre-échange, non mentionné. Quant aux loisirs (qui faisaient l'objet d'un chapitre complet dans le Traité sous la signature de Joffre Dumazedier), ils sont rapportés aux entrées «Front populaire » et " paresse »! Curieusement, toutes les entrées ne s'inscrivent pas comme items dans cet index ; c'est le cas par exemple pour les deux excellents articles, l'un sur le «temps » qui traite des temporalités du travail, et l'autre sur les «territoires » qui souligne l'importance qu'a prise cette notion depuis les années 1980. Sans que cela nuise à l'usage de l'index, il aurait sans doute été assez simple de dresser en fin d'ouvrage une table des matières listant les 141 entrées avec leur page d'accès, ce qui aurait permis au lecteur de se reporter directement à la thématique l'intéressant.

Quelles sont donc ces entrées? On ne saurait prétendre dans cette courte recension analyser l'ensemble des articles dont on doit souligner la remarquable diversité, la solidité des exposés et la grande qualité d'écriture, marquée par sa clarté, quels que soient les auteurs. Si l'on tente quelques regroupements, on note d'abord, bien sûr, que le terme travail est associé directement à plus de quinze entrées telles: « administration du travail », « communauté de travail », « division du travail », etc. Le «travail artistique», le «travail politique» et le «travail social» font l'objet d'un traitement particulier, ce que le Traité ne signalait pas, tout focalisé qu'il était sur le monde industriel. Toutefois, le Dictionnaire ignore la médecine du travail en tant que telle (elle était mentionnée à plusieurs reprises dans le Traité); dans l'index, elle est rapportée à : "cancers professionnels ", "pénibilité ", "santé ", "troubles musculosquelettiques ", mais son action en elle-même, pourtant inscrite historiquement depuis la Libération, et les acteurs qui la pratiquent ne sont pas analysés, alors que, heureusement, l'« inspection du travail » bénéficie d'un article. On peut signaler aussi une entrée originale et bienvenue sur les «écrits de travail » qui offre un éclairage parfaitement inédit sur la question. Si les classes sociales ne font pas l'objet d'une étude, si la population active est inconnue en tant que telle (en entrée comme en index), les "catégories socioprofessionnelles" (notion qui a sa propre entrée) sont largement répertoriées: les "paysans et agriculteurs", les "ouvriers", les " employés ", les « cadres » et les « indépendants » sont soigneusement examinés. Deux entrées sont même consacrées au seul monde patronal : l'une aux "entrepreneurs ", 
l'autre au "patronat». En revanche, la maîtrise qui a accompagné tout le processus d'industrialisation depuis son origine et qui avait toute sa place il y a un demi-siècle dans le Traité, ne fait pas même l'objet d'un item dans l'index thématique. Il est vrai que les directeurs de "ressources humaines» (une entrée) baptisent désormais ces professionnels : managers de proximité...

4 Le Dictionnaire rend compte de l'intérêt des différentes disciplines pour le travail. Certaines sont présentées en tant que telles dans les entrées: la "sociologie", évidemment avec une longue référence aux pères fondateurs de la sociologie du travail, mais aussi l'« histoire », l'« anthropologie », les « technologies de l'information et de la communication", ainsi que l'« ergonomie ». D'autres disciplines sont mentionnées seulement dans leur relation avec l'objet de l'ouvrage. C'est le cas pour l'« économie du travail », le « droit du travail », la "psychologie du travail », la "psychosociologie des organisations ». On a signalé plus haut l'absence de géographes et de démographes parmi les auteurs (si on se réfère à leurs présentations en fin de volume) : on retrouve cette même absence dans la liste des disciplines dont l'analyse est proposée au lecteur. On pourrait aussi s'étonner d'un autre manque, étant donné les forts enjeux sociétaux actuels liés à ce domaine : c'est celui de l'écologie qui n'est répertoriée ni en entrée, ni dans l'index. Le terme environnement auquel on pourrait faire référence n'est mentionné qu'en regard du seul $\mathrm{CHSCT}^{5}$. Le Traité abordait bien l'écologie, mais dans un sens tout différent de l'actuel, puisqu'il s'agissait de l'organisation spatiale, notamment urbaine, et de ses rapports avec les catégories sociales.

5 Si le Dictionnaire se veut accessible, il n'oublie jamais qu'il est une production savante. Aucune des entrées ne se présente comme la simple description du sujet traité. Chacune se réfère à des problématiques charpentées. C'est le cas par exemple de "professions» qui est une synthèse convaincante de l'évolution de ce champ disciplinaire de la sociologie marqué par des controverses depuis son émergence. Ou encore de l'étude des « (systèmes de) relations professionnelles ", largement consacrée à la présentation et la discussion des travaux de John T. Dunlop. Mais certaines entrées sont dédiées spécifiquement à un courant de pensée ou une école théorique. Citons à ce titre l'« économie des conventions ", la "théorie de la régulation sociale ", l'« (école des) relations humaines », ou les " (théories des) relations professionnelles »- qui n'ont aucun équivalent dans l'ancien Traité, même si l'avant-propos faisait mention, en passant, de « certaines différences de jugement ».

6 Délibérément, les directeurs de l'ouvrage ont refusé de faire de ce volume une galerie de portraits. Ils s'en expliquent en indiquant que c'est une entreprise en soi que de rassembler des notices sur ceux qui "ont marqué le travail de leurs empreintes" (p. VIII). Aussi ne livrent-ils que deux entrées biographiques : «Marx Karl, 1818-1883» et «Taylor Frederick Winslow, 1856-1915 » qui, il est vrai, ont marqué chacun à leur manière l'histoire du travail. Cependant, on retrouve dans les différents articles d'autres personnages aussi emblématiques que Condorcet, Frédéric Le Play, Charles Fourier, Emile Cheysson, Henry Le Chatelier ou Elton Mayo. Leurs noms sont également répertoriés dans l'index des noms propres (index nominum) en compagnie des auteurs, sociologues, anthropologues ou économistes, d'autrefois ou contemporains, dont les œuvres sont analysées dans l'ouvrage, index qui rassemble 245 noms. En considérant les entrées qui s'appuient sur leurs travaux, on peut constituer un véritable palmarès. En tête, on y trouvera Karl Marx (dix-sept entrées), puis Georges Friedmann (treize), Émile Durkheim (dix), Adam Smith et Pierre Naville (huit), Max Weber (sept), Michel 
Crozier, Jean-Daniel Reynaud et Alain Touraine (six). Indiquons à ce propos que JeanDaniel Reynaud, qui est aussi un des auteurs du Dictionnaire, était déjà un contributeur du Traité. Quelques rares auteurs des entrées sont signalés dans cet index (sept au total), mais aucun ne l'est pour une des notices qu'il a rédigées.

7 La parcimonie qui a touché les acteurs emblématiques de l'histoire du travail dans le Dictionnaire se retrouve avec les grands faits historiques concernant le monde du travail : seuls deux d'entre eux ont trouvé grâce auprès de l'équipe éditoriale. Il est vrai que ce sont des faits d'importance. Une entrée est dédiée au « Premier Mai », l'autre au «Front populaire ». Mais, pour prendre deux exemples significatifs parmi bien d'autres possibles, la Révolution française qui a tant transformé les rapports de travail et l'encyclique Rerum novarum (1891), à la base de la doctrine sociale de l'Église catholique, ne font l'objet que de brèves mais pertinentes expositions dans la belle entrée " corporatisme "; ce n'est pas faire injure à l'auteur de cet article de penser que ces deux événements auraient pu être traités plus largement dans deux entrées spécifiques.

8 Comme le Traité il y a cinquante ans, le Dictionnaire consacre plusieurs entrées aux activités collectives des travailleurs et aux organismes en liaison avec le travail: "action collective», «communauté de travail», «institutions représentatives du personnel », « organisations internationales du travail », "syndicats, syndicalismes », mais on pourrait tout aussi bien mentionner "militant» ou "négociations", en rapport avec les «conflits» qui supposent des actes de groupe. On retrouverait dans l'index des matières du Traité peu ou prou les mêmes mentions: "cohésion de groupe », " masses syndicales ", " militants », « organisation du travail », « mouvement ouvrier ", etc. La sociologie du travail s'est largement fondée sur l'étude de ces comportements collectifs. Les auteurs du Dictionnaire sont les héritiers de cette tradition et ils ne la renient point. Car les perspectives ont changé. On s'intéresse désormais à d'autres données qui paraissent tout aussi importantes pour comprendre les processus de travail ou ce qui s'y rapporte. Les entrées rendent compte de cette évolution et des travaux accumulés tout au long de ces années, qui prétendent exposer la complexité des phénomènes, mais aussi la perception qu'en ont les individus qui y sont impliqués: "identité », «insertion", "mobilité professionnelle», "performance", "rationalité " sont quelques-uns des articles qui, avec «corps", «émotion » ou " valeur », exposent de façon fine ces transformations et leur prise en considération par les chercheurs.

9 Aussi est-il au bout du compte logique que la production collective des chercheurs en sciences sociales s'intéressant à la question du travail prenne la forme d'un dictionnaire. Pourrait-on aujourd'hui écrire un traité ? Georges Friedmann et Pierre Naville ont beau exposer d'entrée les difficultés recélées par leur entreprise, la complexité des domaines, la mise au point des méthodes, les problèmes jusqu'alors peu ou mal abordés, les problèmes de coordination des connaissances, ils n'en concluent pas moins que leur ouvrage, panoramique et synthétique, était indispensable et que c'est sous la pression de cette nécessité qu'il fut conçu. L'ouvrage se construit, partie après partie, chapitre après chapitre, de façon ordonnée et sereine, pour aboutir à ce Traité auquel les auteurs d'aujourd'hui se réfèrent encore comme à un point de départ structuré, une référence historique, même si c'est pour en montrer les limites, comme il l'est clairement indiqué dans l'entrée "genre» qui expose avec brio le bouleversement soulevé par les analyses exposant, à partir de la fin des années 1980, 
une lecture sexuée des mondes du travail. À mesure que les recherches se sont développées, les grands paradigmes fondamentaux qui organisaient la discipline se sont effrités. Aujourd'hui, il est vain d'espérer, dans un champ aussi large que celui du travail, pouvoir proposer une coordination complète des travaux. Les nouveaux thèmes qui émergent ajoutent à la complexité. L'auteur de l'entrée « pénibilité » explique dès le début au lecteur que, bien que le propre d'un dictionnaire soit de proposer des définitions stabilisées, l'usage de ce terme en vogue « va de pair avec une diversité dans les usages, voire une certaine confusion ». Ce scrupule tout à son honneur pourrait sans doute être exprimé par la plupart des contributeurs du Dictionnaire. Il faut remercier l'équipe éditoriale d'avoir tout mis en œuvre pour que ces conceptions plurielles soient largement exposées et que les nouvelles pistes de recherche soient étudiées au même titre que les données plus arrêtées.

Outre "pénibilité », on trouve en effet dans le dictionnaire plusieurs entrées qui n'étaient pas examinées dans le Traité et qui paraissent caractériser les problèmes contemporains du travail ou, en tout cas, leur prise de conscience par les sociétés d'aujourd'hui : « discrimination », " employabilité », « harcèlement », " précarité » ou encore "troubles musculo-squelettiques». À quoi opposer ces traits négatifs qui participent de la description actuelle du travail? Le Dictionnaire n'est guère encourageant dans ses réponses. En 1962, dans un chapitre particulièrement suggestif du Traité, Jacqueline Frisch-Gautier soulevait le problème du moral et de la satisfaction au travail. Elle revenait notamment sur les travaux d'Henri de Man qui, en 1930, avait examiné la joie au travail, idéalisant quelque peu le travail de l'artiste ou de l'artisan. Elle montrait que le travail était d'abord un moyen de vivre et d'accéder ainsi à des sources de satisfaction et aussi, un lieu de vie important pour qui y passe la moitié de sa vie éveillée, mais qu'il était également source de frustration, aspect défavorable trop aisément oublié. Plus près de nous, Christian Baudelot et Michel Gollac soulevaient la question du bonheur au travail (Travailler pour être heureux? Le bonheur et le travail en France, Paris, Fayard, 2003). La grande enquête qu'ils avaient menée retrouvait l'analyse du Traité quand elle montrait que le travail permettait l'accès à d'autres biens mais que si, en moyenne, pour un Français sur quatre, le travail était une composante importante du bonheur, il fallait examiner la réponse de façon différentielle en tenant compte des catégories professionnelles, de l'âge et du sexe, mais aussi de l'emploi - les chômeurs et les précaires étant les plus nombreux pour qui l'accès à un emploi stable était un préalable à l'épanouissement de soi. Cette thématique n'a pourtant pas été reprise dans une des entrées : parce que la situation actuelle ne justifierait plus de s'y référer? Dans l'index, le terme bonheur renvoie au seul examen des «conditions de travail ", dont le bilan, examiné de façon serrée dans le texte, n'est pas franchement réjouissant, même si l'auteur de la notice, en évoquant Zola, concède que sur le très long terme, ces conditions se sont sans doute quelque peu améliorées... Pour trouver quelque raison d'optimisme, le lecteur du Dictionnaire doit-il seulement s'en remettre à la lecture de l'entrée « utopie»?

11 Mais la consultation de ce dictionnaire, quelle que soit l'entrée à laquelle on l'ouvre, est, elle, un motif permanent de satisfaction. On dispose désormais d'un outil solide, pratique, maniable, qui sera d'un apport constant pour l'enseignant comme pour l'étudiant, pour le syndicaliste comme pour le directeur de ressources humaines, et, plus largement, pour toute personne qui souhaite s'informer et comprendre ce qu'est le travail aujourd'hui dans nos sociétés. 


\section{NOTES}

1. Centre national de la recherche scientifique.

2. Conservatoire national des arts et métiers.

3. École des hautes études en sciences sociales.

4. Institut national de la statistique et des études économiques.

5. Comité d'hygiène, de sécurité et des conditions de travail.

\section{AUTEURS}

\section{ANDRÉ GRELON}

École des hautes études en sciences sociales (EHESS) 